Quorum-sensing contributes to virulence, twitching motility, seed attachment and biofilm formation in the wild type strain Aac-5 of Acidovorax citrulli

Authors: Tielin Wang ${ }^{1,2}$, Wei Guan ${ }^{1}$, Qi Huang ${ }^{3}$, Yuwen Yang ${ }^{1}$, Wanrong Yan ${ }^{1}$, Baixin Sun $^{1}$, Tingchang Zhao ${ }^{1 *}$

\title{
Institutional addresses:
}

${ }^{1}$ State Key Laboratory for Biology of Plant Diseases and Insect Pests, Institute of Plant Protection, Chinese Academy of Agricultural Sciences, Beijing 100193, China.

${ }^{2}$ National Resource Center for Chinese Materia Medica, China Academy of Chinese Medical Sciences, State Key Laboratory Breeding Base of Dao-di Herbs, Beijng, 100700, China.

${ }^{3}$ Floral and Nursery Plants Research Unit, Agricultural Research Service, U. S. Dept. of Agriculture, Beltsvile, Maryland, U. S. A.

*Corresponding author:

Tingchang Zhao, Institute of Plant Protection, Chinese Academy of Agricultural Sciences, Beijing 100193, China. Fax: +86-10-62815933. Email: tingchangzhao@gmail.com

\section{Emails of other authors:}

Tielin Wang: wt182@163.com

Wei Guan: wyngwan@yahoo.com

Qi Huang: qi.huang@ars.usda.gov

Yuwen Yang: yangyuwen@126.com

Wanrong Yan: yanwanrong818@163.com

Baixin Sun: baixin2008@163.com 
Quorum-sensing contributes to virulence, twitching motility, seed attachment and biofilm formation in the wild type strain Aac-5 of Acidovorax citrulli

\begin{abstract}
Acidovorax citrulli is a seed-borne pathogen causing bacterial fruit blotch of cucurbits including melon and watermelon. We investigated the roles of quorum sensing in the wild-type group II strain Aac-5 of A. citrulli by generating aacR and aacI knockout mutants and their complementation strains. We found that twitching motility and virulence were reduced, but biofilm formation and seed attachment were increased significantly in the two mutants as compared to the wild type strain. Deletion of $a a c R$ and $a a c I$, however, had no effect on swimming motility and polar flagella formation of Aac-5. Furthermore, deletion of $a a c R$ resulted in reduced gene expression of $h r p E, h r c N$ and pilT, while deletion of aacI affected only the expression of $h r p E$ and pilT, not $h r c N$.
\end{abstract}

Keywords: Quorum sensing, Acidovorax citrulli, biofilm formation, twitching motility 


\section{Introduction}

Bacteria communicate with each other using a process called "quorum sensing" (QS), which utilizes self-generated signaling molecules termed autoinducers (AIs). N-acyl-homoserine lactone (AHL) is the major class of QS molecules in Gram-negative bacteria, and they are produced by more than 50 different bacterial species [1, 2, 3, 4]. A common method of AHL regulation in Gram-negative bacteria is achieved by two proteins: LuxI, an AI synthase protein and LuxR, a regulatory protein. AI binds to the LuxR family regulators, which subsequently regulate the expression of a wide range of related genes [5]. Traits regulated by QS include the production of extracellular polysaccharides, degradative enzymes, antibiotics, siderophores, and pigments, as well as Hrp protein secretion, Ti plasmid transfer, motility, biofilm formation, and epiphytic fitness $[6,7,8,9]$.

Type III secretion system (T3SS) is a needle like device that bacteria use to deliver type III effector proteins into host cells. The T3SS is encoded by the hrp (HR and pathogenicity) cluster, which includes over 20 genes including hrc (HR and conserved) and hrp genes that encode a type III secretion tunnel [10]. In the hrp cluster, $h r c N$ encodes $\mathrm{HrcN}$, which is a translocase ATPase that is indispensable for T3SS [11]. The $h r p E$ encodes a major component of a surface appendage named the Hrp pilus [12] that is involved in secretion of effector proteins. Type IV pili (TFP) are appendages of bacterial surface that are involved in many processes in bacteria including adherence and colonization, motility, and biofilm formation [13, 14]. The pilT gene encodes an ATPase responsible for TFP retraction that facilitates twitching motility [15]. The pilT deletion mutant strain of Psedomonas aeruginosa completely lost twitching motility [16].

Acidovorax citrulli causes bacterial fruit blotch (BFB) of cucurbits, which results in substantial economic losses to cucurbit growers worldwide [17, 18, 19]. Strains of $A$. citrulli are divided into two groups: group I strains are pathogenic to most cucurbit hosts, and group II strains are comparatively more pathogenic to watermelon seedlings than group I strains [20, 21, 22, 23]. In addition, unlike the group I strains, group II strains are copper sensitive and unable to form biofilms [15]. The role of the QS system in A. citrulli was first studied in the group I strain XJL12 isolated in China [24]. Strain XJL12 was reduced in virulence, growth rate and swimming motility when its aacI (luxI homologue) gene was mutated, but the mutation had no effect on biofilm formation or exopolysaccharide production [25]. A recent study analyzing aacI and aacR, homologues of luxI and luxR, respectively, in the group II strain AAC00-1isolated in the United States found that aacI is involved in seed-to-seedling transmission of BFB in watermelon [26], but biofilm formation was not studied in the study. Since there is no biofilm production in group II strains and it is unknown whether the QS system affects group I and group II strains of A. citrulli differently, we investigated the roles of QS in virulence, twitching and swimming motilities, flagella formation, biofilm formation and seed adhesion ability of a group II strain of A. citrulli 
isolated from watermelon in China [19]. Our results suggest there is a functional difference in the QS systems between the two groups of A. citrulli strains.

\section{Results}

\section{Confirmation of the aacR and aacI mutants and their complementation strains of $\boldsymbol{A}$. citrulli}

The aacR gene encodes the LuxR homologue protein, which is 243 amino acids in length based on the genome sequence of A. citrulli AAC00-1 (GenBank accession number CP000512.1). PCR amplification of the aacR mutant strain with the Rup-F and Rdn-R primers and the subsequent sequencing of the PCR product confirmed that strain $a a c R^{-}$contained a band of $1,918 \mathrm{bp}$, consisting of 515-bp upstream and 548-bp downstream fragments of the aacR gene replaced by an 855-bp gentamicin (Gm) cassette (data not shown). PCR amplification of the aacI mutant strain $a a c I$ with the Iup-F and Idn-A primers and the subsequent sequencing of the PCR product confirmed that strain aacI contained a band of 1,897-bp containing 526-bp upstream and 516-bp downstream fragments of the aacI gene replaced by the 855-bp Gm cassette (data not shown). The presence of the $\mathrm{Gm}$ cassette in $a a c I$ and $a a c R^{-}$was further confirmed by Southern blot and was absent from the wild-type strain Aac5 (data not shown). The $a a c I$ and $a a c R^{-}$strains were stable after continuous culturing for 20 times in KB medium.

The fact that the complementation strains aacR Comp and aacIComp were $\mathrm{Sp}^{\mathrm{R}}$ suggested the successful transfer of the plasmid pHMaacR and pHMaacI into the aacI and aac $R^{-}$strains, respectively. Furthermore, the presence of pHMaacR in aacR Comp was confirmed by PCR using the primer pair aacR-F/R, as the subsequent sequencing of the PCR product showed that the $a a c R^{-}$Comp contained a 1,351-bp fragment including the coding region of $a a c R$ and its promotor region. Similarly, the presence of pHMaacI in aacIComp was confirmed by PCR using the primer pair aacI-F/R, as the subsequent sequencing of the PCR product showed that the aacIComp contained a 1,207-bp fragment including the coding region of aacI and its promotor region.

\section{The aacR and aacI mutants of $A$. citrulli were reduced in twitching motility}

To determine if the QS system has a role in twitching motility in A. citrulli, we compared the wild-type strain Aac-5 to its mutant strains $a a c R^{-}$and $a a c I$ for the formation of corrugated trajectories or halos around their colonies as the bacterium migrating via twitching motility. Strain Aac-5 produced typical haloes, while little or no halos were produced by the aacI and aac $R^{-}$ strains (Fig. 1A). The complemented strains aacR Comp and aacIComp, however, produced similar halos as the wild-type strain Aac-5 (Fig. 1A).

Since TFP are reported to be involved in twitching motility [13, 14, 27, 28], we measured the expression of the TFP gene pilT using quantitative reverse transcription-PCR. Compared to the 
wild-type strain Aac-5, pilT gene expression was reduced 7.7-fold in aacI strain, while little expression was detected in the $a a c R^{-}$strain $(P<0.01)$ (Fig. 1B).

\section{The aacR and aacI mutants of $A$. citrulli were increased in biofilm formation}

Under our qualitative biofilm assay conditions, the wild-type strain Aac-5 did not form any ring of biofilm on the surface of glass flasks, but when the aacR and aacI genes were mutated, the mutant strains $a a c R^{-}$and $a a c I$ formed visible rings of biofilm (Fig. 2A). When the mutant strains were complemented, however, the complementation strains $a a c R^{-C}$ Comp and aacIComp formed little or no visible rings of biofilm (Fig. 2A). This observation was confirmed by our quantitative biofilm assay, as the mean absorption values of the biofilm by $a a c R^{-}$and $a a c I$ were significantly higher than the wild-type strain Aac-5, as well as the mutant complementation strains aacR Comp and aacIComp $(P<0.01)$ (Fig. 2B).

\section{The aacR and aacI mutants of $A$. citrulli increased their ability to adhere to watermelon} seeds

To determine if the QS system in A. citrulli plays a role in the adhesion ability of the bacterium to watermelon seeds, we measured adhesion of A. citrulli strains to watermelon seeds (Fig. 3). The wild type bacterial cells attached to watermelon seeds were estimated to be $8.67 \times 10^{6}$ cells, significantly lower than the two mutant strains (Fig. 3). The aacI was estimated to be $5.87 \times 10^{13}$ cells, the highest among all tested strains (Fig. 3). The mutant complementation strain aacIComp was significantly higher than the wild type strain Aac-5 but lower than the mutant strain aacI, while the mutant complementation strain $a a c R^{-}$Comp was similar to the mutant strain aacR (Fig. 3). To confirm that the difference in seed adhesion was not due to differences in growth ability among tested strains, growth rate was measured for each strain, and no significant differences were observed (data not shown).

\section{The aacR and aacI mutants of $A$. citrulli were reduced in virulence}

Ten days after inoculation, the disease index (DI) in watermelon seedlings caused by the QS mutants $a a c R^{-}$and $a a c I$ were 22.47 and 25.37 , respectively, which were significantly less than the DI of 39.30 caused by the wild-type strain $(P<0.01)$ (Fig. 4$)$. DIs caused by the complementation strains $a a c R^{-}$Comp and $a a c I$ Comp were 37.16 and 35.77 , respectively, not significantly different from the wild-type strain Aac-5 (Fig. 4).

\section{The aacR and aacI mutants did not contribute to swimming motility and flagella biosynthesis of $A$. citrulli}

The halo size as an indication of swimming ability was measured to be $3.07 \mathrm{~cm}, 3.05 \mathrm{~cm}$ and 3.04 $\mathrm{cm}$ in the wild-type Aac-5, and the mutants $a a c R^{-}$and $a a c I$, respectively, not significantly different from each other $(P>0.05)$ (Fig. 5A). In addition, using transmission electron microscopy, 
polar flagella were observed in both of the mutant strains, similar to the ones in the wild-type strain (Fig. 5B).

The aacR mutant of $A$. citrulli were reduced in $h r p E$ and $h r c N$ gene expression, but the aacI mutant only affected $h r p E$ gene expression

The expression level of the T3SS gene hrpE was decreased 5- and 1.4-fold in aacR and aacI mutant strains, respectively, as compared to the wild-type strain Aac-5 (Fig. 6). The expression level of another T3SS gene $h r c N$ was reduced 8.4-fold in the $a a c R^{-}$strain, but was at a similar level in the aacI strain, as compared to strain Aac-5, (Fig. 6).

\section{Discussion}

QS phenomenon is widespread in microbial communities. We investigated the functions of QS in the group II strain Aac-5 of A. citrulli by constructing aacR and aacI mutants and their complementation strains. Our results revealed that $a a c R$ and $a a c I$ positively regulate twitching motility and virulence, negatively regulate the biofilm formation and attachment of the bacterial cells to watermelon seeds, while have no significant effect on polar flagella formation and swimming motility of A. citrulli strain Aac-5.

Twitching is a form of surface motility mediated by TFP $[14,21]$ and is also involved in the initial stage of biofilm formation $[32,33,34,35]$. The TFP of $P$. aeruginosa was shown to have multiple effects on biofilm formation by enabling microcolony formation under some conditions [28, 36]. Similarly, pilT mutants of $P$. aeruginosa have been shown to adhere to different surfaces at similar or higher extents than the wild type [32]. In A. citrulli, strains belonging to group II were previously reported to be unable to form biofilm [15], which was also confirmed in this study. The TFP in A. citrulli was known to be involved in twitching motility [15], and the pilT gene encodes an ATPase responsible for the TFP retraction that drives twitching motility [27]. When the pilT gene was deleted, the mutant exhibited declined twitching motility [15]. In our study, we constructed the QS mutants while keeping the TFP-related genes intact. The twitching motility was reduced significantly in the QS mutants, suggesting that QS regulates twitching motility in $A$. citrulli strain Aac-5, probably through the regulation of the TFP-related genes, including the pilT gene since the expression of this gene was significantly reduced (Fig. 1B). The reduced expression of pilT might also lead to the increased seed attachment by the bacterium to watermelon seeds as revealed by our results (Fig.3), probably due to restrained twitching motility. The aacR and aacI mutant complementation strains did not reduce or only partially reduced seed attachment to the wild type level, suggesting that the $a a c R$ and $a a c I$ genes may need to act in cis for wild type level of seed attachment.

The T3SS is critical to the pathogenicity of gram-negative plant-pathogenic bacteria including $A$. 
citrulli. The hrpE gene in T3SS is essential for the hrp pilus that plays a role in secretion of virulence factors [12]. The $h r c N$ gene encodes the ATPase $\mathrm{HrcN}$, which hydrolyzes ATP in vitro and is essential for T3SS and bacterial pathogenicity [11]. It is therefore not surprising that the deletion of these genes resulted in reduced or even loss of virulence of Aac-5 in both melon and watermelon seedlings [40]. When the aacR and aacI genes were mutated in our study, the virulence of the mutants was significantly reduced, suggesting that the QS contributes to virulence of $A$. citrulli. When the expression of the $h r p E$ gene was measured, its level was significantly reduced 5- and 1.4-fold in the aacR and aacI mutants respectively (Fig. 6), which may result in the reduction in virulence of the mutant strains to watermelon and melon seedlings. When the expression of $h r c N$ was measured, however, its level was significantly decreased in aacR but not in aacI mutant, suggesting a more significant effect of the $a a c R$ gene on the regulation of the T3SS-related gene $h r c N$ than the aacI gene.

In summary, the QS system regulates the twitching motility, virulence, seed adhesion ability and biofilm formation but not flagella formation or swimming motility of the group II strain Aac-5 of A. citrulli. Our result differs from the previous study regarding the group I strain XJL12 of $A$. citrulli in that the biofilm formation of the aacI mutant in XJL12 was similar to the wild-type strain but the growth rate of the mutant was decreased [25], suggesting different effects of aacI on biofilm formation of different groups of A. citrulli strains. Our result is also different from the previous study using the group II strain AAC00-1 isolated in the United States, since no significant difference in seed-to-seedling transmission between the aacR mutant and the wild-type strain AAC00-1 was observed [26], although seed attachment, not seed to seedling transmission was studied in our study. It is possible that the QS system in different groups of A. citrulli strains or strains isolated from different geographical locations may control a particular trait differently, which warrant further studies in the future.

\section{MATERIALS AND METHODS}

\section{Bacterial strains, plasmids and growth conditions}

The bacterial strains and plasmids used in this study are listed in Table 1. A. citrulli wild-type and mutant strains were grown in King's B (KB) broth or KA plate (KB containing agar at $15 \mathrm{~g} /$ liter) with appropriate antibiotics at $28^{\circ} \mathrm{C}$. Escherichia coli strains were grown in Luria-Bertani medium (LB) medium at $37^{\circ} \mathrm{C}$ [41]. The antibiotics used were ampicillin (Ap), kanamycin (Km), spectinomycin $(\mathrm{Sp})$ and gentamicin $(\mathrm{Gm})$ at concentrations of $100 \mu \mathrm{g} \mathrm{mL} L^{-1}$ for Ap and $50 \mu \mathrm{g}$ $\mathrm{mL}^{-1}$ for the other antibiotics.

\section{Construction of the $a a c I$ and $a a c R$ mutants and their complemented strains}

The aacI and aacR genes in the wild-type strain Aac-5 were inactivated by homologous 
integration as described by Windgassen et al. [42], respectively, using the suicide vector pK18mobsacB [43]. Primers for PCR amplification of the two genes, aacR-F/R and aacI-F/R, were designed using the free online program Primer 3.0 (http://www.simgene.com/Primer3) (Table 2). Each reaction mixture contained $0.5 \mu 1$ of DNA template, $6.25 \mu \mathrm{l}$ of $2 \times \mathrm{PCR}$ Mix (TaKaRa, Dlian, China) and $0.5 \mu \mathrm{l}$ of each primer for a total reaction volume of $12.5 \mu \mathrm{l}$. The PCR conditions were $94^{\circ} \mathrm{C}$ for $3 \mathrm{~min}, 30$ cycles of $94^{\circ} \mathrm{C}$ for $30 \mathrm{~s}, 65^{\circ} \mathrm{C}$ for $30 \mathrm{~s}$ and $72^{\circ} \mathrm{C}$ for $90 \mathrm{~s}$, followed by $72^{\circ} \mathrm{C}$ for 5 min. The 1,918-bp fragment amplified from the wild-type strain Aac-5 by the Rup-F and Rdn-R primers (Table 2) contained a 729-bp coding region of the aacR gene, as well as 515and 548-bp upstream and downstream sequences of the gene. The 1,897-bp fragment amplified from Aac-5 by the Iup-F and Rdn-R primers (Table 2) contained a 636-bp coding region of the aacI gene, as well as 526- and 516-bp upstream and downstream sequences of the gene. After confirmation by sequencing, the fragments were digested by EcoRI and HindIII, as well as by HindIII, respectively, and cloned into pK18mobsacB to create plasmids pK18-aacR and pK18-aacI (Table 1). The two plasmids were digested with BamHI and NdeI, and the aacR and aacI gene region were replaced with a $G m$ gene cassette ( $855 \mathrm{bp})$, respectively, to create plasmid pK18-aacRGm and pK18-aacIGm (Table 1). The pK18-aacIGm and pK18-aacRGm were introduced from E. coli DH5 $\alpha$ into Aac-5, respectively, by triparental conjugation using pRK600 as a helper plasmid. Transconjugants were screened on KB supplemented with $10 \%$ sucrose and antibiotics (Ap and Gm) and confirmed by PCR using the Rup-F/Rdn-R and Iup-F/Rdn-R primers, respectively. To confirm the presence of the Gm cassette in the transconjugants, Southern blotting was performed with primers $\mathrm{Gm}-\mathrm{F} / \mathrm{R}$ using marker BM5000 (Biomed, 5,000 bp, 3,000 bp, 2,000 bp, 1,000 bp, $750 \mathrm{bp}, 500 \mathrm{bp}, 250 \mathrm{bp}, 100 \mathrm{bp}$ ) as the probe. The confirmed aacR and aacI mutant strains, $a a c I$ and $a a c R^{-}$(Table 1), was used for subsequent studies.

To generate complementation strains, the $a a c R$ and $a a c I$ genes including their respective promotor region (1,351 bp and 1,207 bp, respectively) in Aac-5 were amplified using primers aacI-F/aacI-R and aacR-F/aacR-R respectively (Table 2). The gene fragments were cloned separately into pHM1 to generate pHM-aacR and pHM-aacI (Table 1), which were transferred into the mutant strains $a a c I$ and $a a c R^{-}$by triparental conjugation, respectively. Transconjugants named aacR Comp and aacIComp were identified through screening on KB (amended with Ap, Sp and Gm) (Table 1). 
All obtained plasmids and A. cirulli strains were confirmed by PCR and DNA sequencing.

\section{Assessment of twitching motility}

Ten microliter suspension $\left(\mathrm{OD}_{600}=1\right)$ of $A$. citrulli strains were placed on KA plus Ap plates and incubated for $72 \mathrm{~h}$, and their twitching motility was visualized using an inverted microscope (Olympus IX83, Japan) according to the protocol of Bahar et al. [15]. Colonies with twitching motility were characterized by the formation of corrugated trajectories or haloes around the colonies.

\section{Virulence assays}

Virulence of the A. citrulli strains were tested on 3-week-old watermelon seedlings (Citrullus lanatus cultivar Jingxin\#6, provided by Beijing Academy of Agriculture and Forestry Sciences, Beijing, China). To prepare bacterial inoculum, A. citrulli strains were grown in KB liquid medium for $24 \mathrm{~h}$, and their $\mathrm{OD}_{600}$ were adjusted with sterile distilled water to 0.6 (approximately $10{ }^{8} \mathrm{CFU} / \mathrm{mL}$ ). Two hundred milliliters of each inoculum was spray inoculated into watermelon seedlings and the disease symptoms were evaluated each day for 10 days after inoculation using a disease index (DI) calculated from a modified 0 to 9 disease severity scale: 0 , no symptoms; 1,3 , 5 and 7, necrotic lesions on approximately 25\%, 50\%, 75\% and 100\% of the cotyledons, respectively; and 9, total death of the seedling. The disease index (DI) was calculated each day based on the following formula:

$$
D I=\sum(A \times B) \times 100 / \sum C \times 9
$$

Where $\mathrm{A}$ is the disease scale $(0,1,3,5,7$ or 9$)$, $\mathrm{B}$ is the number of seedlings in each disease scale, and $\mathrm{C}$ is the total number of seedlings in each treatment. The experiment was repeated three times.

\section{Biofilm assays}

The effect of acI/aacR deletion on biofilm formation was determined qualitatively using glass flasks as described before [45] with modifications. Briefly, overnight cultures of the A. citrulli strains were adjusted to an $\mathrm{OD}_{600}$ of 1.0. One hundred microliters of each cell suspension were transferred into 10 mililiters of $\mathrm{KB}$ broth in glass flasks and incubated at $28^{\circ} \mathrm{C}$ for $7 \mathrm{~d}$ without agitation. The broth was then poured out slowly, and the flasks were rinsed three times with sterile distilled water. After fixating by heating at $80^{\circ} \mathrm{C}$ in an oven (Memmert, Schwabach, Germany) for $30 \mathrm{~min}$, biofilms on the surface of the flasks were stained with $1 \%$ crystal violet for $2 \mathrm{~h}$ at room temperature, followed by three washes with sterile water. A ring of violet precipitation developing on the inner wall of the glass flasks indicated biofilm formation. Biofilm formation was also analyzed quantitatively by solubilizing the stained biofilms with $3 \mathrm{~mL}$ of anhydrous ethanol for 12 $\mathrm{h}$ and measuring the $\mathrm{OD}_{580}$ of the stained suspension with a spectrophotometer (Biophotometer, Eppendorf, Hambug, Germany). The experiment was repeated three times. 


\section{Growth rate assays}

Growth rate of A. citrulli strains was determined as described before [46]. One hundred microliters of A. citrulli strains at $\mathrm{OD}_{600}$ of 0.1 were diluted 1000-fold with $100 \mathrm{ml} \mathrm{KB}$ medium and incubated at $28^{\circ} \mathrm{C}$ with shaking at $180 \mathrm{rpm} . \mathrm{OD}_{600}$ of the suspensions was measured every two hours for 36 hours.

\section{Assay for seed adhesion by $A$. citrulli strains}

The effect of aacI/aacR deletion on seed adhesion was determined using a modified method of Tian et al. [47]. Watermelon seeds were surface sterilized by soaking in $75 \%$ (vol/vol) ethanol twice, each time for $20 \mathrm{~min}$, followed by vigorous rinsing with sterile water for three times. The seeds were air dried and stored at room temperature until use. The seeds were mixed with $10 \mathrm{ml}$ of $10^{3} \mathrm{CFU}$ suspension of each A. citrulli strain with gentle shaking. After incubation for $2 \mathrm{~h}$, the seeds were transferred to a plastic culture tube containing $10 \mathrm{ml}$ of sterile water. The tube was vortexed for $1 \mathrm{~min}$ to remove bacterial cells that were not strongly attached to the seed surface. One seed was then placed into each tube containing $1 \mathrm{~mL}$ of $\mathrm{KB}$, and incubated at $28^{\circ} \mathrm{C}$ for $48 \mathrm{~h}$. Dilution plating on KA plates was done for seed suspensions in each tube to predict relative initial cell adhesion. 10 seeds were used for each treatment and the experiment was repeated at least three times.

\section{Assay for swimming motility and observation of flagella by transmission electron microscopy}

A. citrulli strains were grown in $\mathrm{KB}$, and their $\mathrm{OD}_{600}$ was adjusted to 0.6 . Ten microliters of each bacterial suspension were placed on the center of a soft nutrient agar plate $(0.3 \%$ agar) (basal medium) [15] for $36 \mathrm{~h}$ at $28^{\circ} \mathrm{C}$. The diameter of the colony on each plate was then measured. There were at least 9 plates inoculated by each strain in each experiment, and the experiment was repeated three times.

To observe flagella, each A. citrulli strain was grown on the basal medium. Ten microliters of sterile water were placed on $36 \mathrm{~h}$-old colonies for $2 \mathrm{~min}$, and a 200-mesh formvar-coated copper grid was placed on top of each water drop for $1 \mathrm{~min}$. The copper grids were negatively stained with $1 \%$ uranyl acetate for three time, each time for 30s. Transmission electron microscopy was used to observe polar flagella in each $A$. citrulli strain.

\section{RNA isolation and quantitative reverse transcription (qRT)-PCR analysis of gene expression}

A. citrulli strains were grown in $\mathrm{KB}$ at $28^{\circ} \mathrm{C}$ overnight to an $\mathrm{OD}_{600}$ of 0.8 . The cells were washed twice with sterilized water and used for RNA extraction with the bacterial RNAout kit (Tiandz, Beijing, China) according to the manufacturer's instructions. Extracted RNA was treated with DNase I (New England Biolabs, Beverly, MA, USA) to eliminate any potential DNA 
contaminations. The RNA was then reverse transcripted by using FastQuant RT kit (TianGen, Beijing, China) according to the instructions. Quantitative PCR analysis was carried out with gene-specific primers (Table 3) designed in this study (Table 3) using the same online program as described above. cDNA was used as a template with SYBR Green added in the PCR reaction containing SYBR Supermix (Tiandz, Beijing, China). Relative levels of gene expression were determined using the $2^{-\Delta \Delta \mathrm{CT}}$ method [48], with the glyA gene as the internal control [20]. There were three technical replicates in each experiment and the experiment was repeated three times.

\section{Statistical analysis}

Statistical analysis was performed using the Student's t test in the Excel 2010 software (Microsoft Inc., Seattle, WA, USA). For seed adhesion assay, the number of bacterial cells was analyzed using Duncan's test by using the free tool R 3.1.2. Differences were considered statistically significant if $P<0.01$. 


\section{Acknowledgements}

This research was supported by China's earmarked fund for Modern Agroindustry Technology Research System (CARS-26) and special fund for Agro-Scientific Research in the Public Interest (201003066). We thank Michael J. Stulberg at USDA/ARS in Beltsville, MD, USA for critical review of the manuscript. 


\section{References}

1. M. Safari, R. Amache, E. Esmaeilishirazifard, T. Keshavarz, Microbial metabolism of quorum-sensing molecules acyl-homoserine lactones, $\gamma$-heptalactone and other lactones, Appl. microbiol. and biotechnol. 98(8) (2014), 3401-3412.

2. C. Fuqua, A. Eberhard, Signal generation in autoinduction systems: synthesis of acylated homoserine lactones by LuxI-type proteins, Cell-cell signaling in bacteria, ASM Press, Washington, DC, (1999)211-230.

3. C.M. Waters, B.L. Bassler, Quorum sensing: cell-to-cell communication in bacteria, Annu. Rev. Cell Dev. Biol. 21(2005):319-346.

4. N.A. Whitehead, A.M. Barnard, H. Slater, N.J. Simpson, and G.P. Salmond, Quorum-sensing in Gram-negative bacteria, FEMS Microbiol. Rev. 25(4) (2001):365-404.

5. S.B. Von Bodman, W.D. Bauer, D.L. Coplin, Quorum sensing in plant-pathogenic bacteria, Annu. Rev. Phytopathol. 41(1) (2003): 455-482.

6. M.R. Parsek, E.P. Greenberg, Sociomicrobiology: the connections between quorum sensing and biofilms, Trends in Microbiol. 13 (2005) 27-33.

7. P. Stoodley, K. Sauer, D.G. Davies, J.W. Costerton, Biofilms as complex differentiated communities, Annu. Rev. Microbiol. 56(1) (2002):187-209.

8. L. Crossman, J.M. Dow, Biofilm formation and dispersal in Xanthomonas campestris, Microbes and Infection. 6 (2004) 623-629.

9. R. Daniels, J. Vanderleyden, J. Michiels, Quorum sensing and swarming migration in bacteria, FEMS Microbiol. Rev. 28(3) (2004): 261-289.

10. B.J. Staskawicz, M.B. Mudgett, J.L. Dangl, J.E. Galan, Common and contrasting themes of plant and animal diseases, Science. 292(2001): 2285-2289.

11. C. Lorenz, D. Buttner, Functional Characterization of the Type III Secretion ATPase HrcN from the Plant Pathogen Xanthomonas campestris pv. vesicatoria, J. Bacteriol.191(2009): $1414-1428$.

12. E. Weber, R. Koebnik, Domain Structure of HrpE, the Hrp Pilus Subunit of Xanthomonas campestris pv. vesicatoria, J. Bacteriol. $187 \quad$ (2005) 6175-6186. doi:10.1128/JB.187.17.6175-6186.2005

13. L. Craig, M.E. Pique, J.A. Tainer, Type IV pilus structure and bacterial pathogenicity, Nat. Rev. Microbiol. 2(5) (2004):363-378.

14. J.S. Mattick, Type IV pili and twitching motility, Annu. Rev. Microbiol. 56(1) (2002): 289-314.

15. O. Bahar, T. Goffer, S. Burdman, Type IV pili are required for virulence, twitching motility, and biofilm formation of Acidovorax avenae subsp. citrulli, Mol. Plant Microbe Interact. 22(8) (2009):909-920.

16. J.J. Bertrand, J.T West, J.N. Engel, Genetic Analysis of the Regulation of Type IV Pilus Function by the Chp Chemosensory System of Pseudomonas aeruginosa, J Bacteriol.192(2010): 994-1010. 
17. N.W. Schaad, G. Sowell, R.W. Goth, R.R. Colwell, R.E Webb, Pseudomonas pseudoalcaligenes subsp. citrulli subsp, Int. J. of Syst. Bacteriol. 28(1)(1978): 117-125.

18. N.W. Schaad, E. Postnikova, A. Sechler, L.E. Claflin, A.K. Vidaver, J.B. Jones, B.A. Ramundo, Reclassification of Subspecies of Acidovorax avenae as A. Avenae (Manns 1905) emend., A. cattleyae (Pavarino, 1911) comb., A. citrulli (Schaad et al., 1978) comb., and proposal of A. oryzae sp.,Syst. Appl. Microbiol. 31(6) (2008):434-446.

19. A. Willems, M. Goor, S. Thielemans, M. Gillis, K. Kersters, J. De Ley, 1992. Transfer of several phytopathogenic Pseudomonas species to Acidovorax as Acidovorax avenae subsp. avenae subsp. nov., comb. nov., Acidovorax avenae subsp. citrulli, Acidovorax avenae subsp. cattleyae, and Acidovorax konjaci, Int. J. Syst. Bacteriol. 42(1) (1992):107-119.

20. S. Yan, Y. Yang, T. Wang, T. Zhao, N.W. Schaad, Genetic diversity analysis of Acidovorax citrulli in China, Eur. J. Plant Pathol. 136(1) (2013):171-181.

21. R.R. Walcott, A. Fessehaie, A.C. Castro, Differences in pathogenicity between two genetically distinct groups of Acidovorax avenae subsp. citrulli on cucurbit hosts, J. Phytopath. 152(5) (2004):277-285.

22. S. Burdman, N. Kots, G. Kritzman, J. Kopelowitz, Molecular, physiological, and host-range characterization of Acidovorax avenae subsp. citrulli isolates from watermelon and melon in Israel, Plant Dis. 89(12) (2005):1339-1347.

23. J. Feng, E.L. Schuenzel, J. Li, N.W. Schaad, Multilocus sequence typing reveals two evolutionary lineages of Acidovorax avenae subsp. citrulli, Phytopathology. 99(8) (2009):913-920.

24. T. Chen, G. Qian, X. Yan, 2009. Detection of a quorum sensing signal molecule of Acidovorax avenae subsp. citrulli and its regulation of pathogenicity, Chin. J. Agric. Biotechnol. 6(01) (2009): 49-53.

25. J. Fan, G. Qian, T. Chen, Y. Zhao, F. Liu, R.R Walcott, B. Hu, The acyl-homoserine lactone (AHL)-type quorum sensing system affects growth rate, swimming motility and pathogenicity in Acidovorax avenae subsp. citrulli, World J. Microbiol. Biotechnol. 27(5) (2011): 1155-1166.

26. K.L. Johnson, R.R.Walcott, Quorum Sensing Contributes to Seed-to-Seedling Transmission of Acidovorax citrulli on Watermelon, J. Phytopath. 161(7-8) (2013): 562-573.

27. E. Nudleman, D. Kaiser, Pulling together with type IV pili, J. Mol. Microbiol. Biotechnol. 7(1-2) (2004):52-62.

28. G. O'Toole, R. Kolter, Flagellar and twitchingmotility are necessary for Pseudomonas aeruginosa biofilm development, Mol. Microbiol. 30(1998): 295-304.

29. W.L. Ng, B.L. Bassler, Bacterial quorum-sensing network architectures, Annu. Rev. Genet. 43(2009):197-222.

30. N.R. Stanley, B.A. Lazazzera, Environmental signals and regulatory pathways that influence biofilm formation, Mol. Microbiol. 52(4) (2004): 917-924. 
31. E. Banin,M.L. Vasil, E.P. Greenberg, Iron and Pseudomonas aeruginosa biofilm formation, Proc. Natl. Acad. Sci. USA. 102(31) (2005):11076-11081.

32. M. Klausen, A. Heydorn, P. Ragas, L.Lambertsen, A. Aaes-Jørgensen, S. Molin, T. Tolker-Nielsen, 2003. Biofilm formation by Pseudomonas aeruginosa wild type, flagella and type IV pili mutants, Mol. Microbiol. 48(6) (2003):1511-1524.

33. M. Klausen, M. Gjermansen, J.U. Kreft, T. Tolker-Nielsen, Dynamics of development and dispersal in sessile microbial communities: examples from Pseudomonas aeruginosa and Pseudomonas putida model biofilms, FEMS Microbiol. Lett. 261(1) (2006):1-11.

34. P.K. Singh, Iron sequestration by human lactoferrin stimulates $P$. aeruginosa surface motility and blocks biofilm formation, Biometals 17(3) (2004):267-270.

35. H. Kobayashi, Airway Biofilms, Treat. Respir. Med. 4(4) (2005):241-253.

36. A. Heydorn, B. Ersbøll, J. Kato, M. Hentzer, M.R. Parsek, T. Tolker-Nielsen, S. Molin, Statistical analysis of Pseudomonas aeruginosa biofilm development: impact of mutations in genes involved in twitching motility, cell-to-cell signaling, and stationary-phase sigma factor expression, Appl. Environ. Microbiol. 68(4) (2002): 2008-2017.

37. O. Vidal, R. Longin, C. Prigent-Combaret, C. Dorel, M. Hooreman, P. Lejeune, Isolation of an Escherichia coli K-12 mutant strain able to form biofilms on inert surfaces: involvement of a new ompR allele that increases curli expression, J. Bacteriol. 180(9) (1998):2442-2449.

38. C. Prigent-Combaret, G. Prensier, T.T. Le Thi, O. Vidal, P. Lejeune, C. Dorel, 2000. Developmental pathway for biofilm formation in curli-producing Escherichia coli strains: role of flagella, curli and colanic acid, Environ. Microbiol. 2(4) (2000): 450-464.

39. C. Prigent-Combaret, E. Brombacher, O. Vidal, A. Ambert, P. Lejeune, P. Landini, C. Dorel, Complex regulatory network controls initial adhesion and biofilm formation in Escherichia coli via regulation of thecsgD gene, J. Bacteriol. 183(24) (2001): 7213-7223.

40. W. Yan, T. Wang, Y. Yang, L. Dai, T. Zhao, Biological function analysis of $h r c N$ gene of Acidovorax citrulli, Acta Phytopathol. Sin. 45(1) (2015):33-40.

41. D. Hanahan, Studies on transformation of Escherichia coli with plasmids, J. Mol. Biol. 166(4) (1983): 557-580.

42. M. Windgassen, A. Urban, K. Jaeger, Rapid gene inactivation in Pseudomonas aeruginosa, FEMS Microbiol. Lett.193(2000): 201-205.

43. A. Schafer, A. Tauch, W. Jager, J. Kalinowski, G. Thierbach, A. Puhler, Small mobilizable multi-purpose cloning vectors derived from the Escherichia coli plasmids pK18 and pK19: Selection of defined deletions in the chromosome of Corynebacterium glutamicum, Gene. 145(1994):69-73.

44. O. Bahar, N. Levi, S. Burdman, The cucurbit pathogenic bacterium Acidovorax citrulli requires a polar flagellum for full virulence before and after host-tissue penetration, Mol. Plant Microbe Interact. 24(9) (2011):1040-1050.

45. M.S. Aschtgen, C.S. Bernard, S. De Bentzmann, R. Lloubès, E. Cascales, SciN is an outer 
membrane lipoprotein required for type VI secretion in enteroaggregative Escherichia coli, J. Bacteriol. 190(22) (2008):7523-7531.

46. I Sondi, B. Salopek-Sondi, Silver nanoparticles as antimicrobial agent: a case study on E. coli as a model for Gram-negative bacteria, J Colloid Interface Sci. 275(2004): 177-182.

47. Y. Tian, Y.Zhao, X. Wu, F. Liu, B. Hu, R.R. Walcott, The type VI protein secretion system contributes to biofilm formation and seed-to-seedling transmission of Acidovorax citrulli on melon, Mol. Plant Pathol. 16(2015): 38-47.

48. K.J. Livak, T.D. Schmittgen, Analysis of Relative Gene Expression Data Using Real-Time Quantitative PCR and the $2^{-\Delta \Delta C T}$ Method, Methods, 25(4) (2001), 402-408.

49. R.E. Herman, L.L. McKay, Isolation and partial characterization of plasmid DNA from Streptococcus thermophiles, Appl. Environ. Microbiol. 50(1985): 1103-1106. 
Table 1. Bacterial strains and plasmids used in this study

\begin{tabular}{|c|c|c|}
\hline Strain or plasmid & Description $^{\mathrm{a}}$ & $\begin{array}{l}\text { Reference or } \\
\text { source }\end{array}$ \\
\hline \multicolumn{3}{|l|}{ Strains } \\
\hline \multicolumn{3}{|l|}{ Escherichia coli } \\
\hline DH5 $\alpha$ & $\begin{array}{l}\text { supE44 } \triangle \text { lacU169( } \text { (80lacZ } \triangle \text { M15) hsdR17 recA1 endA1 } \\
\text { gyrA96 thi-1 relA1 }\end{array}$ & {$[41]$} \\
\hline \multicolumn{3}{|l|}{ Acidovorax citrulli } \\
\hline Aac-5 & Wild-type watermelon strain; $A p^{r}$ & {$[20]$} \\
\hline$a a c R^{-}$ & aacR mutant, $\mathrm{Ap}^{\mathrm{r}} ; \mathrm{Gm}^{\mathrm{r}}$ & This study \\
\hline aacI & aacI mutant, $\mathrm{Ap}^{\mathrm{r}} ; \mathrm{Gm}^{\mathrm{r}}$ & This study \\
\hline aacR Comp & $\begin{array}{l}a a c R \text { complementation strain, } a a c R^{-} \text {containing } \mathrm{pHM} a a c R ; \mathrm{Ap}^{\mathrm{r}} \text {, } \\
\mathrm{Gm}^{\mathrm{r}}, \mathrm{Sp}^{\mathrm{r}}\end{array}$ & This study \\
\hline aacIComp & $\begin{array}{l}a a c^{-} \text {complementation strain, aacI containing pHMaacI; } \mathrm{Ap}^{\mathrm{r}} \text {, } \\
\mathrm{Gm}^{\mathrm{r}}, \mathrm{Sp}^{\mathrm{r}}\end{array}$ & This study \\
\hline \multicolumn{3}{|l|}{ Plasmids } \\
\hline pK18mobsacB & $\begin{array}{l}\text { Cloning and suicide vector with a } s a c B \text { gene for mutagenesis; } \\
\mathrm{Km}^{\mathrm{r}}\end{array}$ & {$[43]$} \\
\hline $\mathrm{pK} 18-a a c R$ & $\begin{array}{l}\text { pK18mobsacB carrying } 729-\mathrm{bp} \text { coding region of the aacR gene, } \\
\text { as well as 515- and 548-bp upstream and downstream } \\
\text { sequences of the gene; } \mathrm{Km}^{\mathrm{r}}\end{array}$ & This study \\
\hline pK18-aacI & $\begin{array}{l}\text { pK18mobsacB carrying 636-bp coding region of the aacI gene, } \\
\text { as well as 526- and 516-bp upstream and downstream } \\
\text { sequences of the gene; } \mathrm{Km}^{\mathrm{r}}\end{array}$ & This study \\
\hline $\mathrm{pK} 18-a a c R \mathrm{Gm}$ & $\begin{array}{l}\text { pK18-aacR carrying 729-bp coding region of the aacR gene } \\
\text { replaced by } 855 \text {-bp Gm cassette; } \mathrm{Gm}^{\mathrm{r}}, \mathrm{Km}^{\mathrm{r}}\end{array}$ & This study \\
\hline pK18-aaclGm & $\begin{array}{l}\text { pK18-aacI carrying 636-bp coding region of the aacI gene } \\
\text { replaced by } 855-\mathrm{bp} \mathrm{Gm} \text { cassette; } \mathrm{Gm}^{\mathrm{r}}, \mathrm{Km}^{\mathrm{r}}\end{array}$ & This study \\
\hline pHM1 & Broad-host range expression vector; $\mathrm{Sp}^{\mathrm{r}}$ & [49] \\
\hline pHMaacR & $\begin{array}{l}\text { pHM1 carrying a 1351-bp fragment containing } a a c R \text { and its } \\
\text { promoter; } \mathrm{Sp}^{\mathrm{r}}\end{array}$ & This study \\
\hline pHMaacI & $\begin{array}{l}\text { pHM1 carrying a 1207-bp fragment containg aacI and its } \\
\text { promoter; } \mathrm{Sp}^{\mathrm{r}}\end{array}$ & This study \\
\hline
\end{tabular}

\footnotetext{
${ }^{a} \mathrm{Km}^{\mathrm{r}}, \mathrm{Ap}^{\mathrm{r}}, \mathrm{Gm}^{\mathrm{r}}$, and $\mathrm{Sp}^{\mathrm{r}}$ indicate resistance to kanamycin, ampicillin, gentamycin, and spectinomycin, respectively.
} 
Table 2. Primers used for construction of mutant and complemented strains

\begin{tabular}{llcc}
\hline Primers & Sequence (5'-3', restriction enzyme sites are underlined) & $\begin{array}{r}\text { Restriction } \\
\text { Enzyme }\end{array}$ & $\begin{array}{c}\text { Product } \\
\text { of PCR }\end{array}$ \\
\hline Iup-F & AAGCTTACCAGTTCCGCCACGGTCTGTCCAC & $\begin{array}{r}\text { HindIII } \\
\text { HindIII }\end{array}$ & $1,897 \mathrm{bp}$ \\
Idn-R & AAGCTTCATTGGTGATGAAGCACTTG & EcoRI & $1,918 \mathrm{bp}$ \\
Rup-F & GAATTCAGACGCTCTACGGCACGATA & HindIII & \\
Rdn-R & AAGCTTACCAGTTCCGCCACGGTCTGTCCAC & BamHI & \multirow{2}{*}{$855 \mathrm{bp}$} \\
GmF & GGATCCCGACGCACACCGTGGAAA & SalI & \\
GmR & GTCGACGCGGCG GTGACAATTT & SalI & \multirow{2}{*}{$1,207 \mathrm{bp}$} \\
aacI-F & AAGCTTTTGGGAGGTCGGTACTGAG & HindIII & \\
aacI-R & GTCGACGCAGGATTTTTCTGGCGACCGTGGC & SalI & $1,351 \mathrm{bp}$ \\
aacR-F & AAGCTTGTAGGAAGGGCGGGGGGC & HindIII & \\
aacR-R & GTCGACGCGGGATTGGCATTGGGGG & & \\
\hline
\end{tabular}

Table 3. Primers designed in this study for quantitative reverse transcription PCR

\begin{tabular}{llll}
\hline Primer set & Sequences $(5-3)$ & $\begin{array}{l}\text { Size of PCR } \\
\text { products (bp) }\end{array}$ & Target \\
\hline pilT-F & ATGACGCTGTCCTTCGCCGC & 142 & pilT \\
pilT-R & GGGTGCCGAACACGAGGTGG & & \\
hrcN-F & CGAACACCGAGGGCGGAAAG & 184 & hrcN \\
hrcN-R & TGCTGCTGATGATGGATTCG & & \\
hrpE-F & GTCAGGATGGACACGCAGGC & 110 & hrpE \\
hrpE-R & AACGCATGGTGCTGGCAGAG & & glyA \\
glyA-F & GCGGCAAGTGGTTCAACATC & 139 & \\
glyA-R & GCGATGTCCACCCAGAAGAT & & \\
\hline
\end{tabular}




\section{Figure legend:}

Fig. 1 Comparison of twitching motility in A. citrulli strains under inverted microscopy (A) and by pilT gene expression (B). The wild-type strain Aac-5 and its aacR and aacI mutant complementation strains $a a c R^{-}$Comp and aacIComp produced typical haloes around bacterial colonies, indicating bacterial motility via twitching. The $a a c R$ and $a a c I$ mutant strains $a a c R^{-}$and aacI, however, produced little or no haloes. The pilT gene expression was calculated using the 2 ${ }^{-\triangle \Delta \mathrm{Cq}}$ method [47], and normalized using the expression level of the internal control gene glyA. Error bars represent standard deviations from three biological repeats. $* *: P<0.01$ by the Student's $\mathrm{t}$ test. The experiment was repeated three times with similar results.

Fig. 2 Effect of $a a c R$ and $a a c I$ on biofilm formation in A. citrlli, as visualized by the violet ring formed on the inner surface of the glass flasks (A) and measured under $\mathrm{OD}_{580}$ using stained biofilm solubilized with ethanol (B). The error bar represents standard errors of the means from three experiments, and ** indicates $P<0.01$ by the Student's t test. Aac-5, wild-type strain of $A$. citrulli; aacR, luxR equivalent gene deletion mutant of Aac-5; aacI, luxI equivalent gene deletion mutant of Aac-5; aack Comp, aacR complemented strain of aacR; aacIComp, aacI complemented strain of $a a c I$.

Fig. 3 Effect of aacR and aacI on attachment of A. citrulli strains to watermelon seeds. The amount of bacterial cells attached to watermelon seeds were quantified by dilution plating. The bars represent standard errors of the means from three experiments for each strain. Different letters indicates significant differences $(P<0.05)$ among strains.

Fig.4 Effect of aacR and aacI on A. citrulli virulence on watermelon leaves. Disease index was calculated based on a 0 to 9 disease severity scale of each seedling. The bars represent standard errors of the means from three experiments. ** indicates $P<0.01$ by the Student's $t$ test.

Fig.5 Effect of aacR and aacI on formation of polar flagellum observed under transmission electron microscopy (A) and swimming motility of A. citrulli strains (B). A. citrulli swimming motility was indicated by the formation of haloes with its diameter measured $36 \mathrm{~h}$ after inoculation of the center of each of the soft basal medium plates with each strain of A. citrulli. The bars represent the standard errors of the means from five experiments with nine plates in each experiment for each strain. Polar flagella (indicated by arrows) were observed in all strains of $A$. citrulli including the wild-type strain Aac-5, and the mutant strains aacR ${ }^{-}$and aacr.

Fig. 6. Effect of $a a c R$ and $a a c I$ on the expression of the type III secretion system-related genes $h r p E$ and $h r c N$ in the wild-type strain Aac-5 (mathematically designated as 1) and the mutant strains $a a c R^{-}$and $a a c I$ of $A$. citrulli. Each gene expression was calculated using the $2^{-\Delta \Delta \mathrm{Cq}}$ method [45], and normalized using the internal control gene glyA. Error bars represent standard deviations from three biological repeats. The experiment was repeated three times with similar results. $* *$ indicates $P<0.01$ by the Student's $t$ test. 

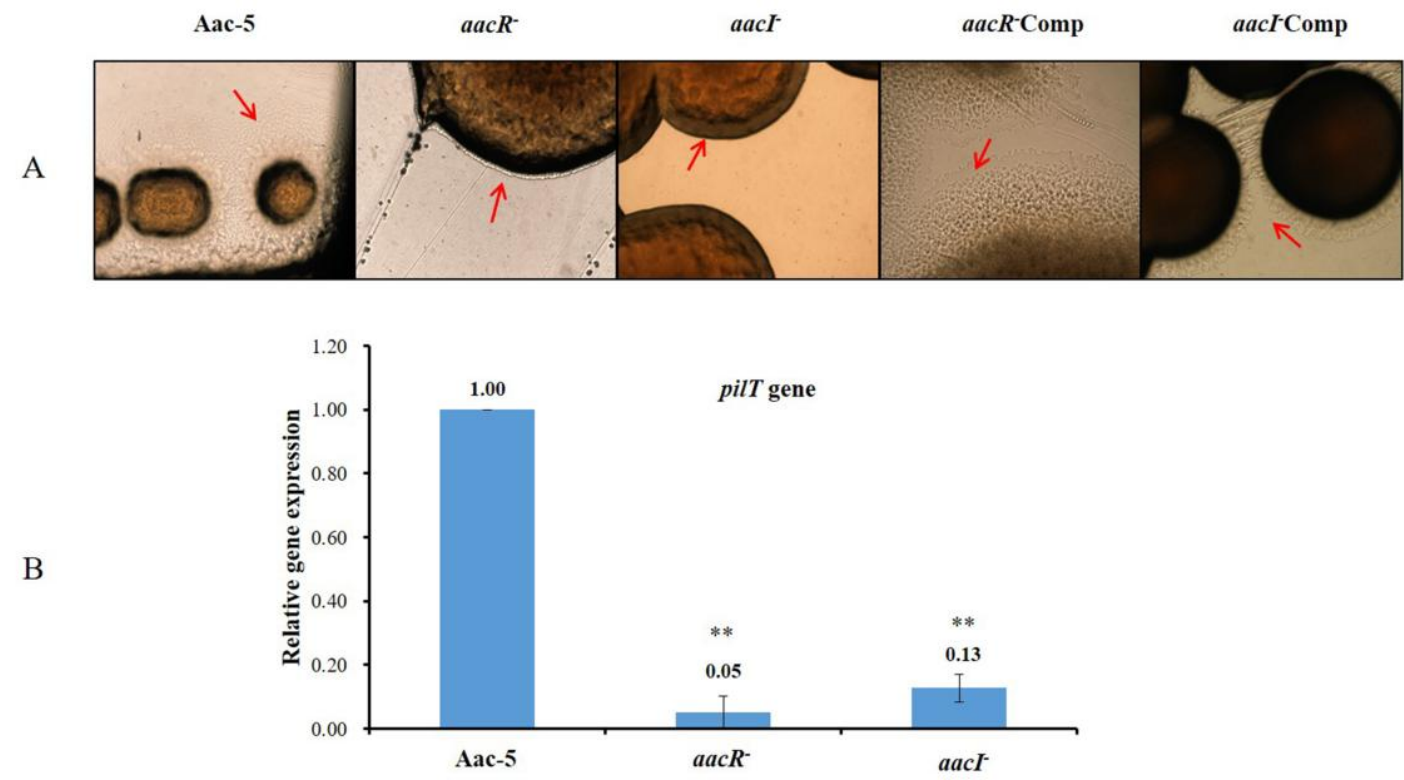


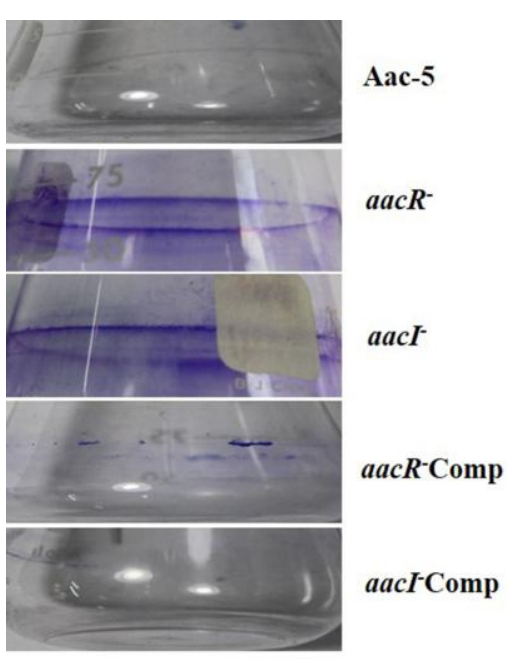

A

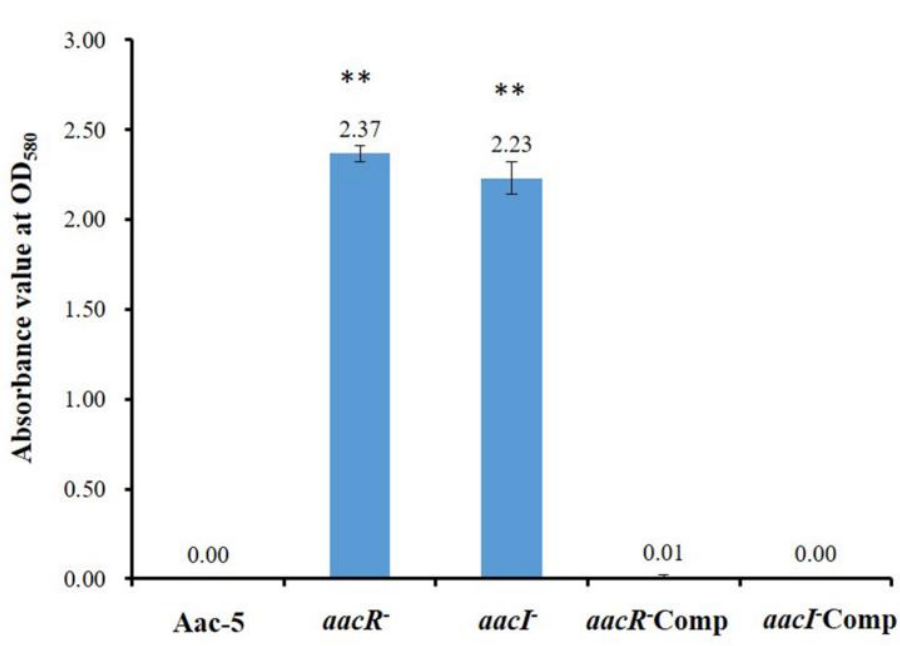

B 


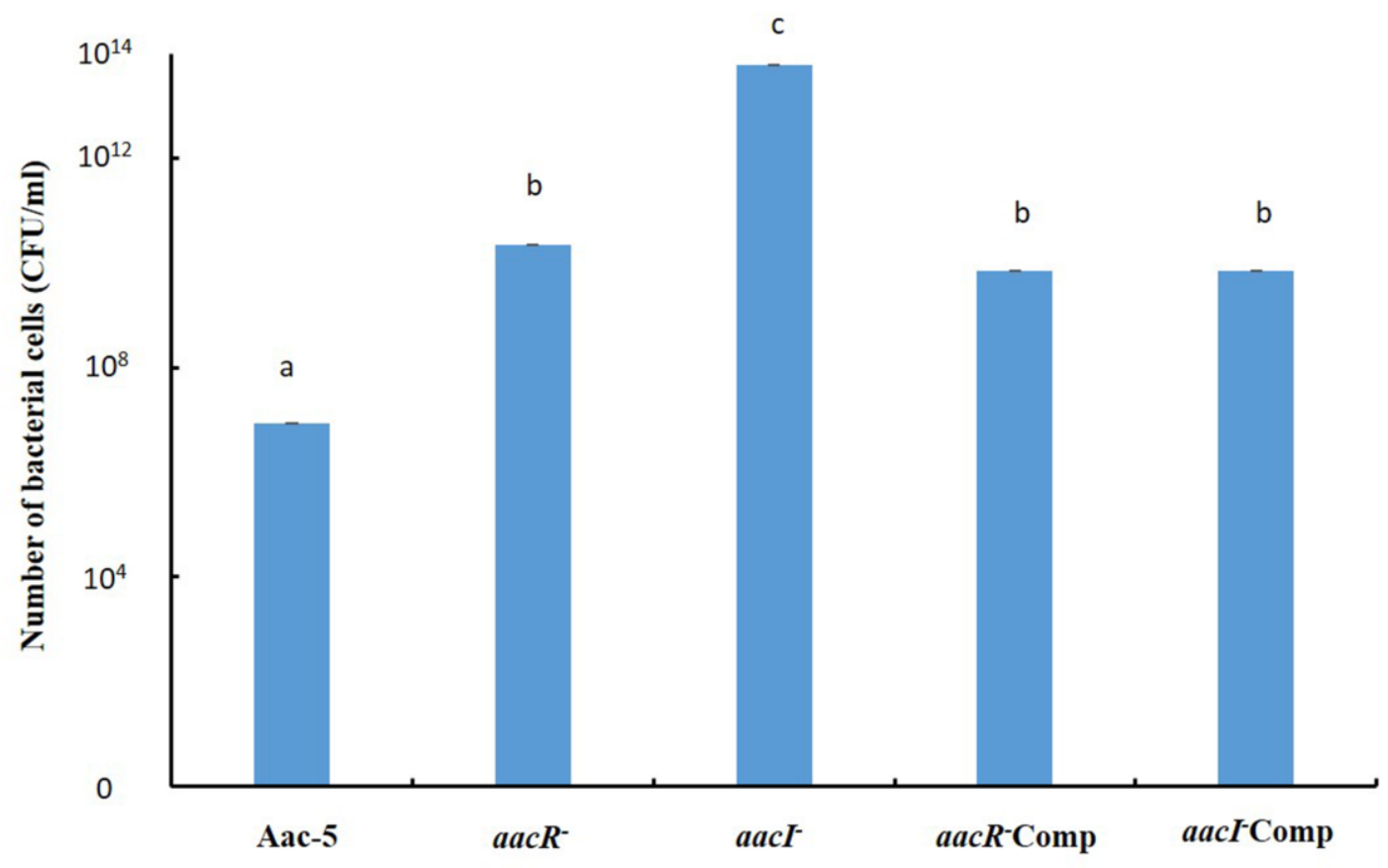




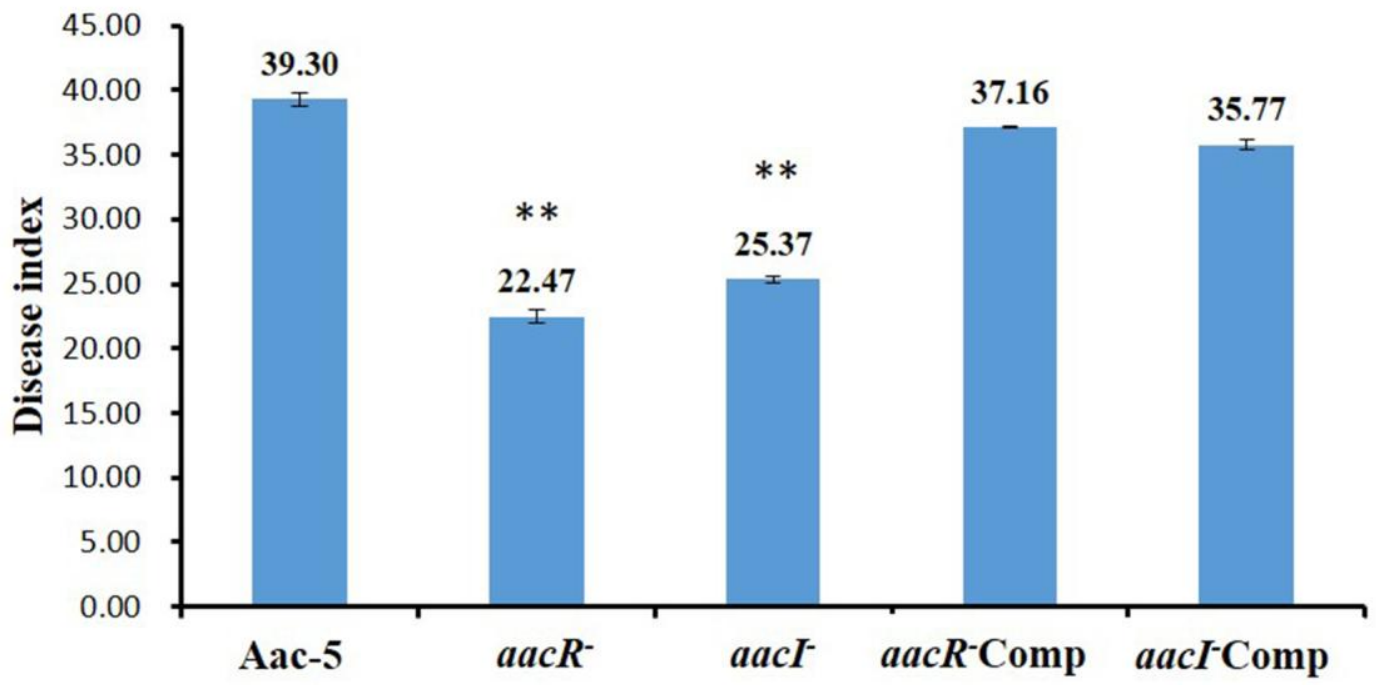


A

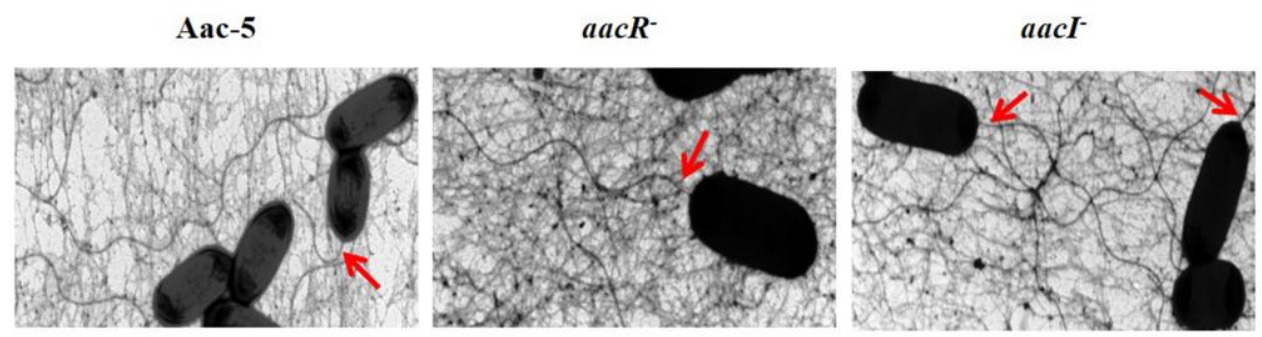

B

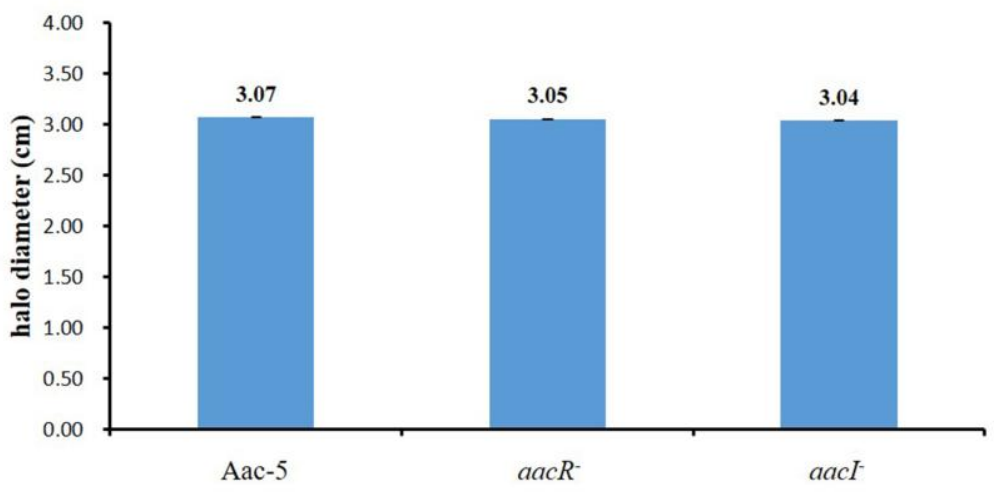




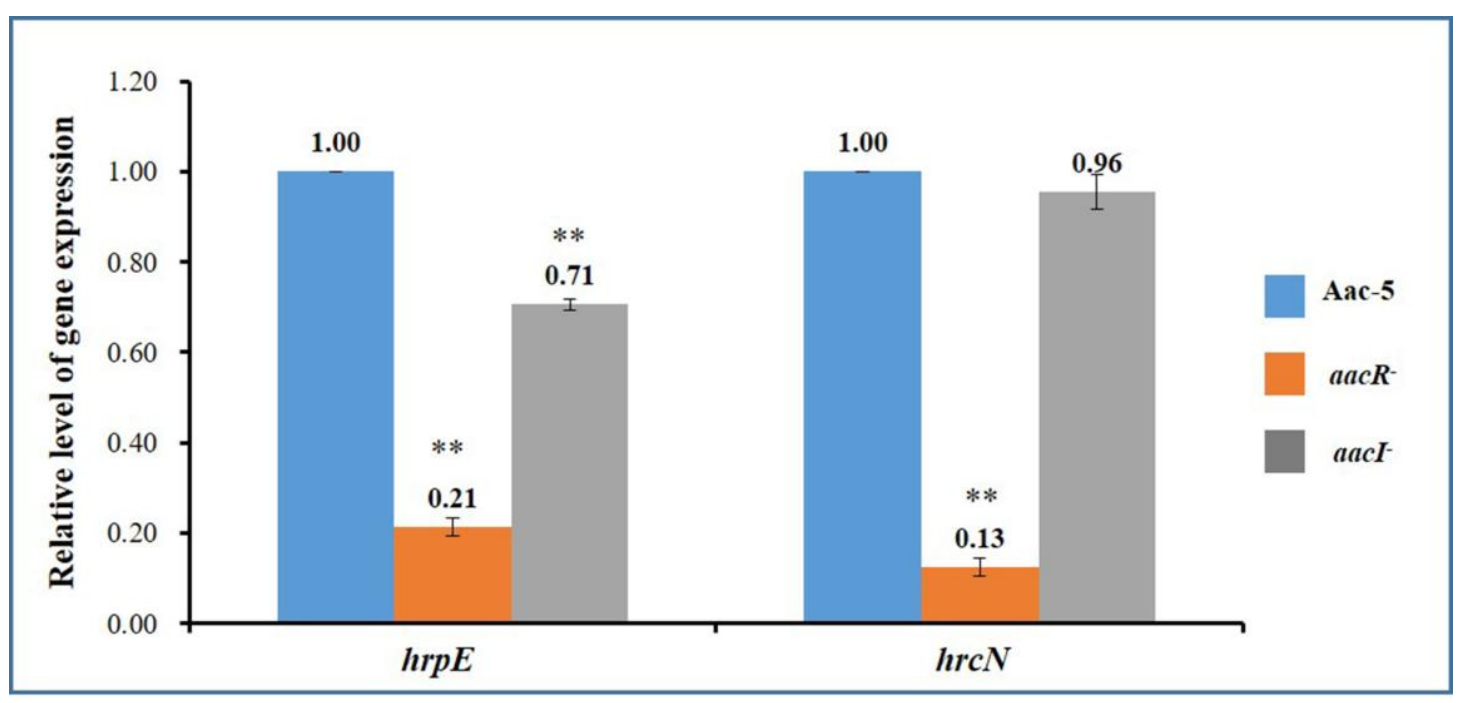

